\title{
THE INFLAMMATORY RESPONSE IN CARDIAC SURGERY. AN UP-TO-DATE OVERVIEW WITH THE EMPHASIS ON THE ROLE OF HEAT SHOCK PROTEINS (HSPs) 60 AND 70
}

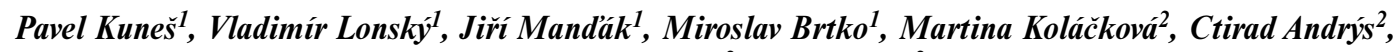 \\ Manuela Kudlová ${ }^{2}$, Jan Krejsek ${ }^{2}$
}

Charles University in Prague, Faculty of Medicine and University Hospital in Hradec Králové, Czech Republic: Department of Cardiac Surgery ${ }^{1}$, Institute of Clinical Immunology and Allergology ${ }^{2}$

Summary: Cardiac surgical operations are associated with the development of a systemic inflammatory response syndrome (SIRS). In most cases, the inflammatory response is apparent only in its subclinical form. In some cases, however, it can become clinically manifest or even life-threatening. This review article presents some new data regarding its biological importance, along with an insight into the recently discovered role played by heat shock proteins 60 and 70 .

Key words: Cardiac surgery; Ischemia and reperfusion; Inflammation; Innate immunity; Heat shock proteins; Lipopolysaccharide; CD14 receptor; Toll-like receptors; Dendritic cells; Lymphocytes

\section{Introduction}

Cardiac surgical operations performed on the open heart are associated with the development of the systemic inflammatory response syndrome (SIRS). In most cases, it is manifested by transient tachycardia, arterial hypo- or hypertension due to dysregulated vasomotion, body temperature rising into the febrile ranges without any sign of concomitant infection, and slight abnormalities in most blood chemistry tests. A subclinical form of SIRS is expressed virtually in all surgical or trauma patients, even in those with relatively mild injuries which actually do not present any therapeutic problem. This mild form of SIRS should be viewed as a beneficial phenomenon, which helps the host to overcome a temporary loss of homeostasis on the whole-body level that has been inflicted by trauma, infection or surgical injury (14). At the same time, this biological response is a prerequisite for proper wound healing and, ultimately, for complete regain or reset of the regulatory homeostatic mechanisms on all organ levels (17). Unrestrained forms of SIRS, which have evaded the network of regulatory mechanisms, develop into exaggerated clinical presentation with deeply pronounced symptoms that have been enumerated above. Ultimately, they can progress into some of the life-threatening manifestations, referred to as the multiple organ dysfunction syndrome (MODS) or the multiple organ failure syndrome (MOFS) (33).

However, to add more complexity to terminological perplexity, there is an alternative classification of the critically ill patients, which meticulously takes into account all slight changes of their individual development. These changes reflect all comorbid conditions, including the degree of their clinical presentation with all available laboratory markers. According to this latter concept, the term of SIRS is characterized by predominance of pro-inflammatory mediators, such as tumor necrosis factor- $\alpha$ and/or interleukin$1 \beta$, right in the initial stages of the critical illness. If the treatment of these gravely affected patients has been successfully conducted, they move on to the next category referred to as MARS. This is an intermediate state with both pro- and anti-inflammatory mediators present in nearly equal amounts. The last clinical presentation is classified as the compensatory anti-inflammatory response syndrome or CARS, with anti-inflammatory mediators clearly predominating while the overall prognosis of patients is improving (21).

\section{Infectious versus non-infectious origin of complications in critically ill patients}

The very reason underlying an unfavorable outcome of critically ill patients is often beyond our understanding, despite all medical and mechanical support expended to maintain their life functions. There are cases in which concomitant infection can be traced as the causative factor. On the other hand, an identical clinical presentation, namely that closely resembling a septic shock syndrome, can be observed in patients without any signs of infection. This latter 
form of SIRS, i.e. the one devoid of infection, is known as the „sepsis-like syndrome“ (15). If mild to moderate form of SIRS, either infectious or not, has moved on to the level of MODS or even MOFS, the function of many organs is heavily compromised or lost. Mortality is high even in wellequipped and well-conducted critical care units. Therefore, any effort aimed at minimizing or eliminating these unfavorable conditions should be taken advantage of, both in the operation room and in the postoperative ward. Irrespective of the ever-growing sum of knowledge in this particular field of medical research, much remains to be understood concerning the natural history of SIRS and consequently, much remains to be learned in order to prevent its full-blown manifestation. Some of the recent findings based on the inflammatory/innate immunity responses with emphasis on the role of heat shock proteins (HSPs) 60 and 70 will be presented in the following text.

\section{Extracorporeal blood circulation in the development of SIRS}

During cardiac operations performed with the use of cardiopulmonary bypass (CPB), alternatively known as „on-pump“ operations, the patient is exposed to extracorporeal circulation and extracorporeal oxygenation of blood. These processes take place in extra-anatomical compartments of the heart-lung machine. In this artificial environment, which is characterized by absence of the endothelial lining, the patient's blood is exposed to altered biophysical forces. Their impact is translated into activation of the plasma humoral cascade systems, i.e. [i.] the complement system, [ii.] the kallikrein-kinin system and [iii.] the coagulation system. At the same time, cellular elements within the blood stream are activated or more properly termed, they are primed. Priming means that they are ready to perform an early-immediate, mostly exaggerated response any time they are challenged by another activatory stimulus (the „second challenge“ or the „two-hit“ model). After returning into the patient's own vascular bed, blood that has been primed in the extracorporeal circuit activates, in turn, his or her endothelial lining. Leukocyte-endothelial cell interactions make up the first step in a sequence of events that culminate in eliciting inflammatory reactions within the interstitial space of many tissues, with the heart and the lungs being the first and the most gravely affected target organs (34).

\section{Endogenous heat shock protein 60}

In parallel to activation or priming, cells of the body set off production of the so-called „danger signal“ molecules. These are endogenous immune stimulators released during unfavorable life conditions in order to alert the immune system to the need for initiating a defense reaction. One of the most prominent „danger signals“ is carried by the heat shock protein 60 (HSP60), the cipher of sixty denominating its molecular weight of $60 \mathrm{kDa}(6)$. Under normal conditions, HSP60 is constitutively produced by all mammalian cells. This constitutive form of HSP60 is operative within the cell interior where it fulfils its „chaperone“ functions. „Chaperoning” means proper folding of newly synthesized proteins, their transport among various intracellular compartments, and/or repairing intracellular proteins, most of them enzymes, which have been damaged by cellular stress. Physiological activity of HSP60 implies that normally this protein does not leave the intracellular milieu. Consequently, HSP60 remains hidden within the cell, out of recognition by the immune system (25). An entirely different situation sets in whenever unfavorable conditions strike a cell or a set of cells within any tissue. A patient undergoing cardiac surgery is an example par excellence of such a condition. His or her HSP60s are increasingly synthesized by endothelial cells, which must face diverse forms of stress, e.g. [i.] altered blood flow, [ii.] oxidative or osmotic stress within the cell(s), [iii.] overwhelming activities of proinflammatory cytokines, or [iv.] penetration of various micro-organisms. HSP60 molecules that have been manufactured in excess are translocated from inside the endothelial cells, in which they are present in conjunction with the mitochondria, onto the cellular surface membrane (24). Cells of manifold origin dying by necrotic death release their own HSP60s into the extracellular space. Whatever the cause of HSP60 being set free, macrophages and dendritic cells, both of them producers of large amounts of intrinsic HSP60, take up additional HSP60 molecules that have been expulsed from their maternal cells. Upon engulfment, HSP60 molecules acquired by macrophages or dendritic cells contribute mightily to the activation of the immune system (30). HSP60 has been found to act as an innate activator of T-lymphocytes or as a mitogen of Blymphocytes (8), whereas the HSP60/LPS complex formed during endotoxemia has been shown to augment the overall activation of innate immunity cells (13). HSP60-influenced B-lymphocytes skew the T-lymphocyte response to a Th0 type, which is characterized by the production of both interferon- $\gamma$ and interleukin-10. By contrast, dendritic cells that have been activated by the HSP60/LPS complex produce high amounts of IFN- $\gamma$ and IL-12 with low levels of IL10, which is a typical Th1-promoting response. All these reactions can be observed during cardiac surgical operations. Under such conditions, severe inflammation can be disseminated throughout the body even in the absence of any circulating bacteria (26).

\section{Exogenous or bacterial heat shock protein 60}

Apart from endogenous HSP60, there are also exogenous forms of HSP60 molecules which are embedded in outer membranes of many micro-organisms, such as Chlamydia pneumoniae, Helicobacter pylori, Porphyromonas gingivalis, Borrelia burgdorferi, Legionella pneumophila, Histoplasma capsulatum or Trypanosoma cruzi, to name only a few. From the evolutionary point of view, members of the 
HSP60 family belong to the most conserved proteins in all living organisms, with as much as $70 \%$ sequence homology found between prokaryotic (microbial) and eukaryotic (human) HSP60 (28). Microbial HSP60s represent typical „pathogen-associated molecular pattern“ molecules (PAMPs), i.e. molecular structures shared by different, often unrelated microbial species. Lipopolysaccharide, the endotoxin of Gram-negative bacteria, is another outstanding example of a PAMP. Peptidoglycan, on the other hand, is the most important PAMP in Gram-positive bacteria (22). All relevant PAMPs, whose occurrence by far exceeds that of mere heat shock proteins, lipopolysaccharide or peptidoglycan, are incessantly screened for by the host via a set of cellular receptors collectively referred to as ,pattern-recognition receptors“ (PRRs) (12). Due to an interplay between the PAMP and PRR networks, it is not necessary for the host to identify right from the beginning all pathogenic micro-organisms at their respective antigenic level. Instead, at this early stage of infection, inflammation or cellular damage, it is both sufficient and efficient for the host's defense machinery to identify the micro-organisms (i.e., the antigens) or altered self-structures (i.e., the neoantigens) via the PRRs, which reliably recognize molecular patterns common to bacteria and mammalian cells (i.e. the PAMPs). In so doing, defense reactions can be started off with ample advance, well before any foreign particles are identified at their individual antigenic levels $(3,18)$.

\section{Heat shock protein 60 and lipopolysaccharide in cellular signaling}

Lipopolysaccharide, the most prominent PAMP in the context of inflammatory/innate immunity reactions, is bound by its target cells by way of the membrane receptor CD14. Cells of monocytic origin are the main population that interacts with lipopolysaccharide. However, endothelial cells and smooth muscle cells of the vascular wall are also equipped with the CD14 receptor, which itself is synthesized by hepatic cells during infection or major surgery, whether cardiac or non-cardiac, as an acute phase reactant (2). Whichever the target cell, LPS is usually attracted by the surface membrane CD14 receptor in conjunction with HSP60. The origin of HSP60 molecules - endogenous (human) versus exogenous (microbial) - is of no significance in the process of danger signaling. The CD14 receptor facilitates the HSP60/LPS complex-induced activation of the immune response both in monocytes/macrophages and in vascular endothelial or smooth muscle cells. However, the distribution of the CD14 receptor is confined to the outer surface of the plasma membrane. In this particular position, the CD14 receptor lacks an intracellular domain, which would be able to transmit the HSP60/LPS complex-borne signal into the intracellular space. Therefore, an additional receptor or a set of receptors is needed to compensate for this drawback by penetrating the entire width of the cellular membrane. This gap in signal trans- mission is filled by the family of Toll-like receptors (TLRs), which themselves are archetypical pattern-recognition receptors. Out of this receptor family, which up to now counts at least ten members, Toll-like receptor 4 stands out as the most important LPS-binding PRR (7). The intracellular domain of TLR4 is closely reminiscent of corresponding domains of receptors for interleukin (IL)-1 and IL-18. Hence their collective denomination of TIR, which designs „the Toll/interleukin-1 receptor“ (4).

Macrophages and dendritic cells, main cellular actors in innate immunity responses, use TLR4 signaling to transmit the HSP60/LPS-borne signal, whereas dendritic cells themselves are also responsive to TLR2. B-lymphocytes, by contrast, respond to HSP60 via TLR4.

The HSP60/LPS complex has been found to induce maturation of dendritic cells. This process is characterized by up-regulation of costimulatory molecules:

i. CD40, a basic costimulatory molecule indispensable for dendritic cell maturation,

ii. MHC II (major histocompatibility complex class II),

iii. B7-2, a costimulatory molecule important for Th2 responses,

iv. CD54 or ICAM-1 (intercellular adhesion molecule-1).

In responding to HSP60, B-lymphocytes up-regulate their expression of:

v. CD40,

vi. MHC II,

vii. B7-2,

viii. CD69, an early activation marker.

B-lymphocytes, however, do not up-regulate the expression of ICAM-1 (CD54).

All in all, the effects of the HSP60/LPS complex on dendritic cells and the effects of HSP60 on B-lymphocytes promote the capacity of these cells to act as antigen-presenting cells (APCs). This is an outstanding example of innate immunity passing over to acquired immunity responses (27). T-lymphocytes, main cellular actors in acquired immunity reactions, respond to HSP60 via TLR2 with no involvement of TLR4. This particular response, surprisingly at the first glance, down-modulates T-lymphocyte-mediated immune reactions. Net result of TLR2-mediated HSP60 influence on T-lymphocytes is an inhibition of secretion of proinflammatory cytokines INF- $\gamma$ and/or TNF- $\alpha$ on the one hand, and an increase of secretion of anti-inflammatory cytokines TGF- $\beta$ and/or IL-10 on the other hand. The ensuing T-lymphocyte response is then a diminution of cellular chemotaxis, signaling and proliferation (35).

\section{Intracellular processes leading to SIRS}

To sum up what has been said so far, the external signal initiated by the HSP60/LPS complex enters the intracellular space by way of the CD14/TIR receptor complex. Once within the intracellular milieu, this signal is transmitted further by way of even more complex enzymatic cascades, all of which converge on the level of the transcription fac- 


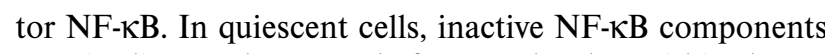
remain dispersed separately from each other within the cytoplasm. After the cell has been stimulated, an active form of this transcription factor is made by assembling all its individual components. Thereafter, the active form of NF- $\kappa B$ is moved from cellular plasma to the nucleus. Here, transcription of the genetic information from nuclear DNA to messenger RNA is started off. Under the directory supervision of the transcription factor NF- $\mathrm{KB}$, this process leads to de novo synthesis of proinflammatory cytokines, chemokines, adhesion molecules and growth factors. All these newly formed peptides participate actively in the development of the inflammatory response, both on the local and on the systemic levels. On the systemic level, one of the clinical presentations resulting from the impact of the transcription factor NF- $\kappa \mathrm{B}$ on proteosynthesis may be the syndrome of SIRS. This syndrome may be synonymous either with the „sepsis syndrome“ in cases of bacterial infection or with the „sepsis-like syndrome“ in cases of sterile inflammation. The latter is pertinent to cardiac surgical patients, even if they do not develop any overt peri- or post-operative complications (1).

\section{SIRS in cardiac surgery}

Viewed as basically useful, SIRS and SIRS-like reactions have been conserved during evolution so that the host might survive in an unfriendly environment, such as the strenuous exercise of the „fight or flight“ nature, multiple injuries or burns, infections or, more recently, any major surgery. Irrespective of the underlying cause, tight control of every step of the inflammatory processes must be exerted both on local and on systemic levels. The control network involves activities of the neuro-immune, the endocrine and the circulatory systems. If the control fails, morbidity and mortality increase dramatically. Conceivably, these defense reactions have not developed in response to cardiac surgery, and even less do they respond specifically to cardiopulmonary bypass surgery. The final form and/or extent of SIRS in cardiac surgical patients are fine-tuned by conditions relevant to this specific procedure. These conditions can be divided into three closely interrelated steps (23).

\section{Contact activation of the blood}

The patients' blood is temporarily diverted into an extra-anatomical vascular bed, i.e. the tubing system of the heart-lung machine. Endothelial lining is absent in this circuit and, accordingly, the latter is devoid of any trace of the anti-adhesive, anti-inflammatory and cytoprotective cellular layer offered by endothelial cells. Contact of blood with this artificial material invariably results in activation or priming of blood-borne cellular elements, notably white blood cells and platelets, and in activation of humoral immune/inflammatory cascades, such as the complement system, the kallikrein-kinin system and the coagulation system. Plasma bactericidal capacity increases progressively even in the absence of any circulating bacteria due to activation of bactericidal enzymes, such as myeloperoxidase, elastase and phospholipase A2. Overwhelming production of „proximal" inflammatory cytokines TNF- $\alpha$, IL- $1 \beta$ and IL- 6 , all of which set in motion the inflammatory response, elicits with a short delay in time the synthesis of a huge wave of chemokines, such as IL-8, MCP-1 or RANTES. Chemokines in their turn support migration and accumulation of activated leukocytes in inflammatory foci. At the same time, synthesis of acute-phase reactants is set off with C-reactive protein (CRP) and pentraxin-3 (PTX3) predominating. In the setting of full-blown SIRS, both of the latter peptides lose their original protective properties, while they gain an overall damaging capacity. Both CRP and PTX3 activate the complement system via the alternative pathway. Ongoing production of anaphylatoxin C5a and the terminal complement complexes C5b-9(n) contributes mightily to the damaging effects of cardiopulmonary bypass. Inflammatory cytokines elicit mainly activation of the vascular endothelium. Endothelial cells' properties are converted from an anti-adhesive and anti-inflammatory phenotype into a procoagulant and pro-inflammatory one, with unrestrained expression of adhesion molecules and coagulation factors. At the same time, synthesis of endogenous anti-inflammatory peptides, such as IL-10 and TGF- $\beta$, which in normal conditions would satisfactorily counter-balance the damaging activities of their pro-inflammatory antipodes, is substantially reduced. The blood-gas interface of the artificial oxygenator, whose pro-inflammatory capacity is of extraordinary magnitude, supplies yet an activatory stimulus to all blood cellular elements and humoral cascades. The extent of their activation is directly proportional to the duration of aortic cross-clamping, which elicits global myocardial ischemia, and to the extracorporeal circulation of blood. However, it must be emphasized that the genetic background of each individual patient plays an important, if not decisive, role in all these processes, whose impact on the outcome of SIRS is now increasingly recognized (19).

\section{2a. Myocardial ischemia and reperfusion: an overflow of inflammatory mediators}

Global myocardial ischemia in CPB patients is induced by cross-clamping the ascending aorta. After the surgical procedure on the arrested heart has been terminated, aortic cross-clamp is released and reperfusion of the myocardium sets in. Reoxygenation of any ischemic tissue unchains a complex array of inflammatory events. Some of the proinflammatory signaling cascades have been set in motion during the period of oxygen deprivation by way of transcription factors HIF-1 $\alpha$ (hypoxia-inducible factor) and Egr-1 (early growth response). With reintroduction of oxygen-rich blood, the heart is overflowed by inflammatory cytokines, mainly IL- 6 and TNF- $\alpha$. Interleukin- 6 is a typical product of ischemic myocardial tissue. These „proximal“ 
cytokines induce up-regulation of endothelial adhesion molecules, such as ICAM-1 (intercellular adhesion molecule), VCAM-1 (vascular cell adhesion molecule) and/or E-selectin. Their emergence on the endothelial surface sets the stage for leukocyte-endothelial interactions, which are closely followed by transmigration of activated neutrophils and monocytes into the interstitial space of the reperfused tissue. This is true not only for the heart but also for the lungs, whose perfusion during extracorporeal circulation of blood has been interrupted, with only bronchiolar arterioles remaining perfused. The latter, however, belong to the systemic circulation. As such, they do not participate in blood gas exchange. The interstitial space of reperfused organs is reminiscent of a battle-field, in which activated neutrophils attack virtually any live cell within their reach. It is of no importance whether the targets are the host's own cells that have been so far happy to survive the period of oxygen deprivation, or whether they are pathogenic microorganisms that must be disposed of as soon as possible. The cytotoxic armamentarium of activated neutrophils is guaranteed by reactive oxygen species (ROS), formerly known as oxygen free radicals. Among them, the superoxide radical $\mathrm{O}_{2}^{-}$is a relatively moderate weapon, whereas the hydroxyl radical $\cdot \mathrm{OH}$, by contrast, is an extremely aggressive oxidant. During myocardial reperfusion, ROS generated by activated neutrophils attack cardiac muscle cells which are prone to die by necrotic or apoptotic death. Cardiac muscle cells that have resisted free radical attack have been successful only at the cost of limiting their metabolic activities and biomechanical performance next to zero. Thus, many muscle cells in the reperfused/reoxygenated myocardium of cardiac surgical patients are by now stunned or even dead (5).

The extent of damage inflicted by leukocyte-derived ROS depends to a great extent upon the quality of myocardial protection achieved during perioperative cardiac arrest and continuing thereafter. This protection may rely upon endogenous protective mechanisms within the heart itself, which help cardiac cells to put up with the sequelae of the „double-edged sword“ of myocardial reperfusion. Apart from anti-oxidant enzymes, which will not be discussed in this text, there is an important, albeit not yet entirely understood, protective role played by the heat shock proteins, notably HSP70.

\section{2b. Genesis and presumed activities of HSP70 in the reperfused heart}

HSP70 is expressed in many organs, including the heart. To make the HSP70 issue yet more complex, it should be pointed out that the rough denomination of „HSP70“ includes at least three members of this protein family, namely:

1. the constitutive form of HSP73, alternatively known as HSC70,

2. the stress-inducible form of HSP70, also referred to as HSP72, which will be the leading topic of the following text.
3. Recently, an additional HSP70-like protein 1 (HSP70L1) has been discovered. It is a dendritic cell-derived protein, which skews immune responses in favor of the Th1 pathway. Its molecular weight of $54.8 \mathrm{kDa}$ being somewhat smaller than that of classical HSP70 molecules, this protein all the same resembles other members of this family both structurally and functionally.

Evidence is accumulating that HSP70 might play an important role in the protective mechanisms which seek to conserve both cardiac function and cellular metabolism in the setting of ischemia/reperfusion or hypoxia/reoxygenation-induced injury (31). Under conditions of cellular stress, HSP70 is translocated from the cytosol to the nucleus, where it interacts with the chromatin-binding enzyme known as the poly(ADP-ribose) polymerase. This is a sensor enzyme which is converted to its active form in the process of recognizing nuclear DNA single-strand breaks and, at the same time, it participates in the repair of damaged DNA. However, as is often the case in biological systems, this same enzyme is also suspected to play an active role in the process of reperfusion injury. Whenever cellular oxygen is replenished, activation of poly(ADP-ribose) polymerase can exceed actual metabolic demands. Its exaggerated activation in the reperfused and reoxygenated heart will consume large amounts of the cellular content of ATP and $\mathrm{NAD}^{+}$. The result will be energy starvation, which would mostly strike cells that have just survived the stress of oxygen deprivation. Accordingly, poly(ADP-ribose) polymerase inhibitors might repair damage inflicted to the hearts in vivo, with rapid postischemic recovery of cardiac function. It has been shown that an untoward increase in the poly (ADP-ribose) polymerase activity in hypoxic/reoxygenated myocardial cells can be diminished by heat shock treatment, which results in HSP production. Maximal attenuation of this potentially deleterious enzyme is achieved after 12 hours of heat exposure. Among various heat shock proteins that are thus generated, HSP70 is the most important one as far as cardiac protection is concerned. HSP70 can efficiently attenuate unfavorable poly(ADP-ribose) polymerase activation.(16).

An alternative cytoprotective and prosurvival capacity of HSP70 under stress conditions might reside in its capacity to inhibit lysosomal membrane permeabilization. Originally, lysosomes were considered "suicide bags“ that caused autolysis in the process of tissue damage through the release of unspecific enzymes during uncontrolled or poorly controlled inflammatory/innate immunity reactions. Accumulating data show that lysosomes also function as death signal integrators in many well-controlled death processes. Cytotoxic lysosomal proteases and cathepsins leave the lysosomal lumen into the cytosol in response to a variety of cellular death stimuli, such as TNF- $\alpha$, Fas, p53 activation and/or oxidative stress. Once released to the cytosol, cathepsins - especially cysteine cathepsins B and L and aspartyl cathepsin D - may trigger cellular death featuring apoptosis- or necrosis-like morphology. By stabilizing lysosomal membranes, HSP70 
can effectively rescue cells form apoptotic or necrotic death induced by proinflammatory cytokines, notably TNF- $\alpha$, or oxidative stress, both of them active contributors to myocardial ischemia-reperfusion injury. The almost universal prosurvival capacity of HSP70 has also got its less friendly face - „the hidden side of the moon” - which is exhibited by many tumor cells. The latter can thus escape a timely and definitive removal from the maternal tissue. Tumor growth may thus go on in an unrestricted manner (20).

\section{2c. HSP70 and its activities in cardiac surgery}

Whenever myocardial damage exceeds the intrinsic cytoprotective capacity of HSP70, HSP70 protein is released from the intracellular compartment to the extracellular space. This is also the case in patients with acute myocardial infarction (9). Just the same as HSP60, the former protein comes now into contact with the immune system, from whose screening activities it is normally hidden. HSP70 activates immune cells by way of the CD14 membrane receptor, TLR-2 and/or TLR-4, and its signal is transmitted to the cellular interior through the MyD88-IRAK-NF- $\kappa \mathrm{B}$ cascade. In the end, translocation of the transcription factor NF- $\kappa \mathrm{B}$ from cellular plasma to the nucleus occurs (32). After cardiac surgery, extracellular HSP70 is regularly detected. This means that some, albeit negligible cardiomyocyte death must have occurred despite all precautions taken. Peak HSP70 levels are attained as soon as two hours after the beginning of surgery. They correlate closely with the time of aortic crossclamping and the duration of extracorporeal circulation. As has been shown by Brit Dybdahl and coworkers, patients operated on without the use of CPB, i.e. „off-pump“ patients, release as little as one fourth of the HSP70 content found in „on-pump“ patients. It should be underscored that both groups of patients release extracellular HSP70 even during wholly uncomplicated peri- and post-operative course. The main difference between the groups is the magnitude of the released protein. Free HSP70 levels correlate tightly both with troponin T and creatine kinase MB isoenzyme levels if some damage to the heart has been inflicted. There is also a strong correlation between HSP70 and IL-10 levels, which is, however, pronounced only in „on-pump“ patients, with their „off-pump“ counterparts releasing practically no extracellular IL-10. There is also an important correlation between HSP70 and IL-6 levels, this particular one irrespective of the type of operation (10). The extent of IL-6 levels has been found to be related predominantly to the extent of surgical trauma. On the other hand, IL- 6 has been proved to be produced directly within the ischemic heart in cardiac surgical and myocardial infarction patients.

From what has been said so far, it can be inferred that CPB may have some negative impact of its own on the heart. The target cells of extracellular HSP70 are predominantly monocytes, which are activated via the CD14-TLR4 pathway (29). Net result of these interactions is a support to inflammatory responses, even in strictly sterile condi- tions. In order for the host to counteract inappropriate inflammation, its principal mediators, i.e. the CD14 receptor protein on the monocyte membrane including both the TLR-2 and the TLR-4 receptors, are down-regulated after surgery. Later on, in the following 24-48 hours, the same proteins are either returning to normal or even up-regulated, which implies monocyte activation in this time frame after surgery. This is also in keeping with our own results, (manuscript in preparation).

\section{Transient endotoxemia due to splanchnic hypoperfusion}

Transient endotoxemia is the result of insufficient blood supply to the splanchnic vascular bed after a substantial amount of blood volume has been excluded from the patient's own vasculature into the tubing circuit of the heartlung machine. Relative hypoperfusion of the splanchnic vascular bed elicits gut wall ischemia, with subsequent increase of villous capillary permeability and translocation of lipopolysaccharide, i.e. the endotoxin of Gram-negative bacteria, or even the patient's own intestinal flora into the systemic circulation. Scavenging capacity of the hepatic reticuloendothelial system, namely the Kupffer cells, which normally set up a reliable barrier against bacterial transition from the portal vein into systemic circulation, is rapidly overcome. Splanchnic hypoperfusion cannot be completely prevented even by ample substitution of blood volume using blood transfusions, much less so by way of crystalloid and/or colloid fluid resuscitation. One reason for this failure can be set down to the steady laminar blood flow generated by the roller pumps of the heart-lung machine instead of the pulsatile blood flow generated with each contraction of the patient's own heart. According to the latest knowledge, LPS can be detected even in the blood stream of otherwise healthy individuals; furthermore, its concentrations can be measured with ultra-sensitive laboratory methods. Ischemic increase of gut permeability only accentuates this phenomenon. LPS concentrations in peripheral blood can increase in such a measure which allows concomitant entry of loose extracellular HSP60 in the absence of any bacterial infection. One intriguing question is whether the patient's own commensal flora, which undoubtedly can supply large amounts of HSP60, should be regarded as undergoing temporary conversion from symbiosis to infection. Whichever is the case, it seems plausible to hypothesize that in cardiac surgical patients, whether or not surgery has been performed with the use of CPB, the ability of endogenous HSP60 to bind circulating LPS and to present the HSP60/LPS complexes to the CD14/TLR2/TLR4 signal transmission system is enhanced in such an extent that starts off one of the first steps in the development of postoperative SIRS. Additional conditions, the list of which is beyond the scope of this article, thereafter make the difference between clinically silent and clinically manifest SIRS. The latter is endowed with the capacity to progress to its life-threatening forms (11). 


\section{Conclusion}

Inflammatory/innate immunity reactions elicited by cardiac surgery and, in a broader sense, by any disruption of bodily integrity, serve the host to overcome these unfavorable conditions and to return to his or her previous well-being. If the inflicted damage cannot be resolved without functional or morphological sequelae, the inflammatory reactions try to minimize any resulting deficit. It is one of the paramount biological paradoxes that the same inflammatory/innate immunity reactions themselves can substantially deepen nearly all initial damage. Heat shock proteins have been recently recognized to form an integral part of these defense reactions. Their uniqueness is underscored by the fact that HSPs play comparable if not superimposed roles both in sterile inflammation, such as that seen in surgical patients, and in septic inflammation, either in primary infection or in infectious complications of originally aseptic processes.

\section{Acknowledgements}

This work has been supported by a research program of The Czech Ministry of Youth, Scholarship and Physical Activities No. MSM 0021620812 and Internal Grant Agency of Ministry of Health, Czech Republic, No. NR/9090-4.

\section{References}

1. Anselmi A, Abbate A, Girola F et al. Myocardial ischemia, stunning, inflammation, and apoptosis during cardiac surgery: a review of evidence. Eur J CardioThorac Surg 2004;25:304-11.

2. Bas S, Gauthier BR, Spenato U et al. CD14 is an acute-phase protein. J Immunol 2004;172:4470-9.

3. Bethke K, Staib F, Distler M et al. Different efficacy of heat shock proteins (HSP) to activate human monocytes and dendritic cells: superiority of HSP60. J Immunol 2002;169:6141-8

4. Beutler B, Hoebe K, Georgel P et al. Genetic analysis of innate immunity: TIR adapter proteins in innate and adaptive immune responses. Microbes Infection 2004;6:1374-81

5. Boyd JH, Mathur S, Wang Y, Bateman RM, Walley KR. Toll-like receptor stimulation in cardiomyocytes decreases contractility and initiates an NF- $\mathrm{KB}$ dependent inflammatory response. Cardiovasc Res 2006;72:384-93.

6. Chen W, Syldath U, Bellmann K et al. Human 60-kDa heat-shock protein: a danger signal to the innate immune system. J Immunol 1999;162:3212-19.

7. Chow JC, Young DW, Golenbock DT et al. Toll-like receptor 4 mediates lipopolysaccharide-induced signal transduction. J Biol Chem 1999;274:10689-92.

8. Cohen-Sfady M, Nussbaum G, Pevsner-Fischer M et al. Heat shock protein 60 activates B cells via the TLR4-MyD88 pathway. J Immunol 2005;175:3594-602.

9. Dybdahl B, Slordahl SA, Waage A, Kierulf P, Espevik T, Sundan A. Myocardial ischemia and the inflammatory response: release of heat shock protein 70 after myocardial infarction. Heart 2005;91:299-304.

10. Dybdahl B, Wahba A, Haaverstad R et al. On-pump versus off-pump coronary artery bypass grafting: more heat shock protein 70 is released after on-pump surgery. Eur J Cardio-Thorac Surg 2004;25:985-92.

11. Fujiyoshi N, Feketova E, Lu Q, Xu D-Z, Haskó G, Deitch EA. Amiloride mode- rates increased gut permeability and diminishes mesenteric lymph-mediated priming of neutrophils in trauma/hemorrhage shock. Surgery 2006;140:810-17.

12. Gordon S. Pattern recognition receptors. Doubling up for the innate immune response. Cell 2002;111:927-30.

13. Habich C, Kempe K, van der Zee R et al. Heat shock protein 60: specific binding of lipopolysaccharide. J Immunol 2005;174:1298-1305.

14. Hackett CJ. Innate immune activation as a broad-spectrum biodefense strategy. Prospects and research challenges. J Allerg Clin Immunol 2003;112:686-94.

15. Johnson GB, Brunn GJ, Platt JL. An endogenous pathway to systemic inflammatory response syndrome (SIRS)-like reactions through Toll-like receptor 4. J Immunol 2004;172:20-24.

16. Kawana K-i, Miyamoto Y, Tanonaka K et al. Cytoprotective mechanism of heat shock protein 70 against hypoxia/reoxygenation injury. J Mol Cell Cardiol 2000; 32:2229-37.

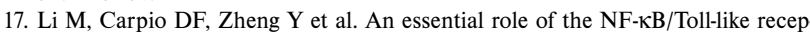
tor pathway in induction of inflammatory and tissue-repair gene expression by necrotic cells. J Immunol 2001;166:7128-35.

18. Lien E, Means TK, Heine $\mathrm{H}$ et al. Toll-like receptor 4 imparts ligand-specific recognition of bacterial lipopolysaccharide. J Clin Invest 2000;105:497-504.

19. Majetschak M, Krehmeier U, Ostroverkh L, Blömeke B, Schäfer M. Alterations in leukocyte function following surgical trauma: differentiation of distinct reaction types and association with tumor necrosis factor gene polymorphisms. Clin Diag Lab Immunol 2005;12:296-303.

20. Nylandsted J, Gyrd-Hansen M, Danielewicz A et al. Heat shock protein 70 promotes cell survival by inhibiting lysosomal membrane permeabilization. J Exp Med 2004;200:425-35.

21. Osuchowski MF, Welch K, Siddiqui J, Remick DG. Circulating cytokine/inhibitor profiles reshape the understanding of the SIRS/CARS continuum in sepsis and predict mortality. J Immunol 2006;177:1967-74.

22. Ozinsky A, Underhill DM, Fontenot JD et al. The repertoire for pattern recognition of pathogens by the innate immune system is defined by cooperation between Toll-like receptors. Proc Natl Acad Sci USA 2000;97:13766-71.

23. Paparella D, Yau TM, Young E. Cardiopulmonary bypass induced inflammation: pathophysiology and treatment. An update. Eur J Cardio-Thorac Surg 2002; 21:232-44.

24. Pfister G, Strohl CM, Perschinka H, et al. Detection of HSP60 on the membrane surface of stressed human endothelial cells bz atomic force and confocal microscopy. J Cell Sci 2005;118:1587-94.

25. Pockley AG. Heat shock proteins as regulators of the immune response. Lancet 2003;362:469-76

26. Quintana FJ, Cohen IR. Heat shock proteins as endogenous adjuvants in sterile and septic inflammation. J Immunol 2005;175:2777-82.

27. Robert J. Evolution of heat shock protein and immunity. Develop Compar Immunol 2003;27:449-64.

28. Stewart GR, Young DB. Heat shock proteins and the host-pathogen interaction during bacterial infection. Curr Opin Immunol 2004;16:506-10.

29. Thériault JR, Mambula SS, Sawamura T, Stevenson MA, Calderwood SK. Extracellular HSP70 binding to surface receptors present on antigen presenting cells and endothelial/epithelial cells. FEBS Lett 2005;579:1951-60.

30. Vabulas RM, Ahmad-Nejad P, da Costa C et al. Endocytosed HSP60s use Tolllike receptor 2 (TLR2) and TLR4 to activate the Toll/interleukin-1 receptor signaling pathway in innate immune cells. J Biol Chem 2001;276:31332-9.

31. Vogt S, Portig I, Kusch B, et al. Detection of anti-hsp 70 immunoglobulin G antibodies indicates better outcome in coronary artery bypass grafting patients suffering from severe preoperative angina. Ann Thorac Surg 2004;78:883-9.

32. Warger T, Hilf N, Rechtsteiner G et al. Interaction of TLR2 and TLR4 ligands with the $\mathrm{N}$-terminal domain of $\mathrm{Gp} 96$ amplifies innate and adaptive immune responses. J Biol Chem 2006;281:22545-53.

33. Weigand MA, Hörner C, Bardenheuer HJ, Bouchon A. The systemic inflammatory response syndrome. Best Pract Res Clin Anaesth 2004;18:455-75.

34. Zahler S, Massoudy P, Hartl $\mathrm{H}$ et al. Acute cardiac inflammatory responses to postischemic reperfusion during cardiopulmonary bypass. Cardiovasc Res 1999; 41:722-30.

35. Zanin-Zhorov A, Tal G, Shivtiel S et al. Heat shock protein 60 activates cytokineassociated negative regulator suppressor of cytokine signaling 3 in T cells: effects on signaling, chemotaxis, and inflammation. J Immunol 2005;175:276-85.

Submitted April 2007. Accepted May 2007.

\section{Corresponding author:}

Pavel Kuneš, M.D., University Hospital Hradec Králové, Department of Cardiac Surgery,

Sokolská 581, 50005 Hradec Králové, Czech Republic, e-mail: kunes.pavel@fnhk.cz 